\title{
Effects of Interaction in the Hofstadter regime of the honeycomb lattice
}

\author{
Archana Mishra, S. R. Hassan, and R. Shankar \\ The Institute of Mathematical Sciences, C.I.T. Campus, Chennai 600 113, India
}

(Dated: February 19, 2018)

\begin{abstract}
We investigate phases of spinless fermions on the honeycomb lattice with nearest neighbor interaction in the Hofstadter regime. The interaction induces incompressible nematic and ferri-electric phases with broken translation symmetry. Some of the transitions are accompanied by changes in the Hall conductivity. We study pair correlations and show that the quantum metric, averaged over the Brillouin zone, characterizes the shape of the pair correlation function.

PACS numbers: 71.10.Fd, 71.27.+a, 71.30.+h
\end{abstract}

\section{INTRODUCTION}

Two dimensional electron systems in a periodic potential in the presence of a magnetic field are characterized by two length scales, the periodicity of the potential and the magnetic length. The regime where these two length scales are comparable exhibits very interesting phenomena like the Hofstadter butterfly ${ }^{1}$. Recently this regime has been accessed experimentally with observations of a stable Hofstadter spectrum in graphene superlattices ${ }^{2-6}$ and realization of the Hofstadter Hamiltonian in cold atoms systems ${ }^{7,8}$. This has motivated us to investigate the effects of repulsive interactions in this regime. The interactions are expected to induce charge ordering in the ground state which may spontaneously break the translational symmetry of the system. Consequently, one of the length scales, namely the periodicity, can change. This could change the fractal structure. Therefore, we investigate translational symmetry breaking in this system. Interaction induced translational symmetry breaking phases have been studied in the honeycomb lattice in the absence of magnetic field ${ }^{9-11}$. Effects of interactions on the Hofstadter butterfly have been discussed previously ${ }^{12-16}$; these works do not consider translational symmetry breaking.

We study a system of spinless fermions on the honeycomb lattice in the Hofstadter regime with nearest neighbor repulsive interactions using mean field theory. We restrict ourselves to the cases when the Fermi level lies in a gap and thus expect the mean field approximation to be good. The ground state of the non-interacting system has all the symmetries of the Hamiltonian and non-zero Hall conductivity. Our studies reveal various interesting phases as the strength of the interaction is increased. We get a first order Landau transition to a phase with broken translational and rotational symmetries. This phase is an incompressible nematic phase characterized by an electric quadrupole moment of the ground state charge distribution. The transition is accompanied by a change in the Hall conductivity. In some cases, on further increasing the interaction, there is another first order Landau transition to an incompressible ferri-electric phase where the inversion symmetry is also broken and the system develops an electric dipole moment.
Nematic phases in homogeneous quantum Hall systems have previously been theoretically studied ${ }^{17-20}$ and experimentally observed ${ }^{21-23}$ in fractional quantum Hall systems. Our results show that they occur in the Hofstadter regime also.

The nematic order parameter has been related to the quantum metric ${ }^{18,20}$. This motivates us to investigate the structure of the anisotropic phases by studying pair correlations from the point of view of the quantum geometric approach to insulating states ${ }^{24}$. We find that the shape and the extent of the pair correlations can be exactly related to the quantum metric in the momentum space $^{24}$, averaged over the Brillouin zone $(\mathrm{BZ})^{25}$. This result relates the momentum space quantum metric of systems in a periodic potential to the real space metric introduced by Haldane ${ }^{18}$ in homogeneous quantum Hall systems.

The remaining part of the paper is organized as follows. We discuss the interacting Hofstadter Hamiltonian and its symmetries in section II. The noninteracting physics of this model and the band topology is illustrated here. The mean field approximation used to solve the interacting Hamiltonian is described in section III. We give explicit description of the complex phases and the phase transitions obtained from solving the self consistency equations in section IV. In section V, we discuss the geometry of the ground state and give a relation between pair correlation function and the quantum metric. We summarize and discuss the results in section VI. The Appendices A and $\mathrm{B}$ contain the description of Chern numbers at half filling and the energy band diagrams in the symmetric and nematic phases respectively.

\section{MODEL AND SYMMETRIES}

The model Hamiltonian we study is,

$$
H=-t \sum_{\langle i j\rangle}\left(c_{i}^{\dagger} e^{i \frac{e}{\hbar} A_{\langle i j\rangle}} c_{j}+h . c\right)+V \sum_{\langle i j\rangle} n_{i} n_{j},
$$

where $c_{i}\left(c_{i}^{\dagger}\right)$ is the annihilation (creation) operator for electrons at site $i$ on the honeycomb lattice, $n_{i}$ is the number density operator, $t$ is the nearest neighbor hopping parameter and $V$ is the nearest neighbor interac- 
tion strength. We consider $t=1$ and $V$ is in units of the hopping matrix element. $A_{\langle i j\rangle}$ are the gauge fields on the nearest neighbor links such that the magnetic flux passing through each plaquette is $\phi=\frac{1}{q} \frac{h}{e}$ where $q$ is an integer.

For these flux values, it has been shown ${ }^{26}$ that the noninteracting theory has three regimes of electron densities. The dilute limit is called the Fermi regime where each filled band has Hall conductivity, $\sigma_{H}=-e^{2} / h$. Thus, $\sigma_{H}=-m e^{2} / h$ when $m$ bands are filled as is the case for non-relativistic systems in the continuum. At a certain filling, $m^{*}$, there is a band with a very large Chern number where the Hall conductivity changes sign when it is completely filled. This band lies in the energy region of the van Hove singularity of the system in the absence of the magnetic field. We refer to the $m^{*}$ filling as the van Hove filling. Pairs of bands get degenerate (at large $q$ ) on further filling. The Hall conductivity changes in steps of $2 e^{2} / h$ when the Fermi level lies in the gap. This is called the Dirac regime. Fig. 1 shows the plot for Hall conductivity vs Fermi energy showing the three different regimes for $q=30$. The blue line is for the Fermi regime and the red line is in the Dirac regime.

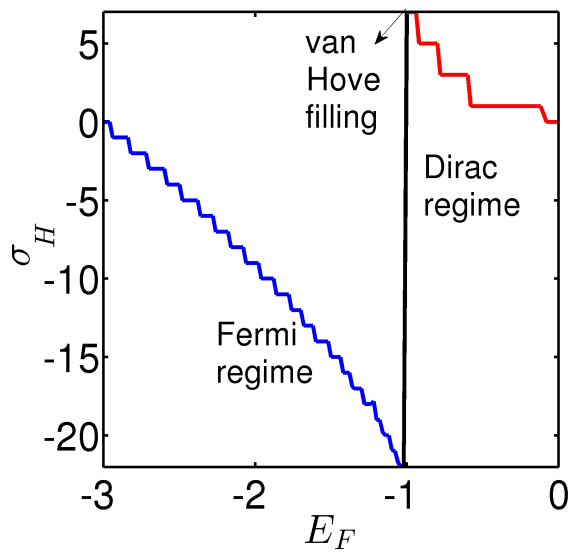

FIG. 1: (Color online) Hall conductivity vs Fermi energy plot for $q=30$. The Fermi regime, van Hove filling and Dirac regime are shown in this figure.

In this paper we concentrate on $q=3$ which is the simplest case where, in the non-interacting system, the bands are well separated with energy gaps, except for the middle bands which touch at 6 Dirac points. The Chern numbers of the $m=1,2$ and 3 bands are $(-1,2,1)$. Consequently, the Hall conductivities of the system with one, two and three bands filled are $\sigma_{H}=-e^{2} / h, e^{2} / h$ and 0 respectively. Going by the behavior of the large $q$ systems discussed above we refer to these three fillings as the Fermi, van Hove and Dirac fillings.

The Hamiltonian is invariant under magnetic translations, $\tau_{1}$ and $\tau_{2}$ which are along $\hat{e}_{1}$ and $\hat{e}_{2}$ directions respectively shown in Fig. 2. These magnetic translation operators do not commute with each other, $\tau_{1} \tau_{2} \tau_{1}^{-1} \tau_{2}^{-1}=e^{i \frac{2 \pi}{3}}$. Thus we need to choose a magnetic unit cell consisting of three original unit cells to implement the Bloch theory. There are two ways of doing this: the linear unit cell denoted as unit cell choice $\mathbf{I}$ with basis vectors $3 \hat{e}_{1}, \hat{e}_{2}$ as shown by the rectangular region shaded in gray in Fig. 2 and the hexagonal unit cell denoted as unit cell choice II with basis vectors $\hat{e}_{2}-\hat{e}_{1}, \hat{e}_{2}+2 \hat{e}_{1}$ as shown by the hexagonal region shaded in yellow in Fig. 2. Unit cell choice II is a rotational symmetric choice where each sublattice is surrounded with three different sublattices unlike unit cell choice $\mathbf{I}$.

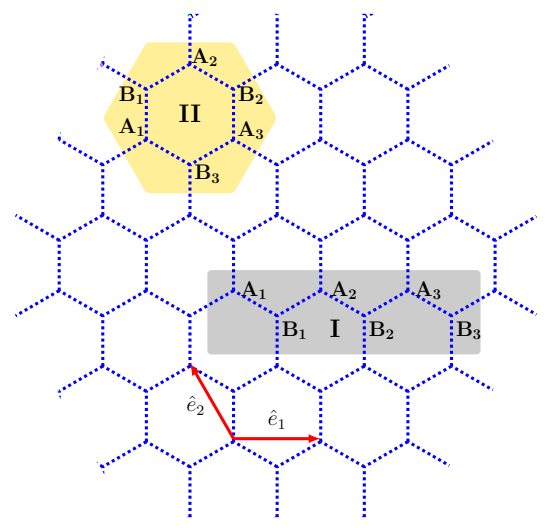

FIG. 2: (Color online) Honeycomb lattice in a magnetic field with flux $\phi=\frac{h}{3 e}$ passing through each plaquette. $A$ and $B$ are the two sublattices. We consider two possible choices of unit cell: linear choice (I) shown as a gray rectangle and hexagonal choice (II) shown as a yellow hexagon. $\left(A_{1}, B_{1}, A_{2}, B_{2}, A_{3}, B_{3}\right)$ are sublattices of these magnetic unit cells. $\hat{e}_{1}$ and $\hat{e}_{2}$ represent the basis vectors of the original lattice.

The Hamiltonian is also invariant under three fold rotations about the sites and two fold rotations about the center of the links. We refer to the two-fold rotation symmetry as inversion symmetry. At half filling, the system also has particle-hole (chiral) symmetry, $c_{i} \rightarrow(-1)^{p_{i}} c_{i}^{\dagger}$, where $p_{i}=0$ for $i$ belonging to one of the sublattices and $p_{i}=1$ for the other.

\section{MEAN FIELD APPROXIMATION}

As mentioned earlier, we analyze the system using mean field theory. The mean field decomposition we use is $^{11}$,

$$
\begin{aligned}
n_{i} n_{j} & \approx\left(\Delta_{i} c_{j}^{\dagger} c_{j}+\Delta_{j} c_{i}^{\dagger} c_{i}\right)-\chi_{\langle i j\rangle} c_{i}^{\dagger} c_{j}-\chi_{\langle i j\rangle}^{*} c_{j}^{\dagger} c_{i} \\
& -\frac{1}{V}\left(\Delta_{i}^{2}+\Delta_{j}^{2}-\left|\chi_{\langle i j\rangle}\right|^{2}\right), \\
\frac{1}{V} \chi_{\langle i j\rangle} & =\left\langle c_{j}^{\dagger} c_{i}\right\rangle_{M F}, \quad \frac{1}{V} \Delta_{i}=\sum_{j(i)}\left\langle c_{j}^{\dagger} c_{j}\right\rangle_{M F}
\end{aligned}
$$

where $j(i)$ denotes all the nearest neighbors of $i$. The self consistency equations (3) have to be solved keeping the number density fixed. 
The single particle mean field Hamiltonian in momentum space is the $6 \times 6$ matrix $h_{M F}(\vec{k})=h_{0}(\vec{k})+$ $h_{F}(\vec{k}, \Delta, \chi)$ where $h_{0}$ is the single particle non-interacting part of the Hamiltonian and $h_{F}$ represents the interaction with the mean field parameters; $\vec{k}$ takes values in the reduced (magnetic) BZ. There are 6 real charge density order parameters represented by $\Delta_{(\alpha, a)}$ and 9 complex bond order parameters represented by $\chi_{\langle(\alpha, a)(\beta, b)\rangle}$ where $\alpha, \beta$ label the original unit cells in the magnetic unit cell and $a, b$ label the two sublattices of the honeycomb lattice. Our mean field ansatz allows the breaking of the translational, rotational and inversion symmetries of the system.

We solve the self consistency equations, Eq. (3), for $\Delta$ and $\chi$ for both choices of the unit cell, I and II. These correspond to different mean field ansatze. The complex bond order parameters $\chi_{\langle(\alpha, a)(\beta, b)\rangle}$ and real charge order parameters $\Delta_{\alpha, a}$ are solved by an iterative method using the self consistency equations, Eq. (3), at a given filling and $V$. We summarize the algorithm used here. (i) We start the iteration with a random initial guess of $\chi_{\langle(\alpha, a)(\beta, b)\rangle}$ and $\Delta_{\alpha, a}$, (ii) diagonalize $H_{M F}$ using $\chi_{\langle(\alpha, a)(\beta, b)\rangle}$ and $\Delta_{\alpha, a}$, (iii) Tune the chemical potential by fixing the number of particles (iv) calculate the expectation value of the link operators and the number operators on each site in the magnetic unit cell. Using this we compute the new values of $\chi_{\langle(\alpha, a)(\beta, b)\rangle}$ and $\Delta_{\alpha, a}$ from Eqs. (3). The whole process from step (ii) to (iv) is repeated until all the quantities converge. We repeat this process for various initial guesses and often find different mean field solutions. Comparing the energies of these solutions, we pick up the lowest energy state as the ground state of the interacting Hamiltonian. This method is repeated for $V \in(1,10)$ for $m=1,2,3$ and we get various complex phases which are discussed in the following section.

\section{RESULTS}

This section describes various phases which are the minimum energy solutions of the self consistency equations. For small $V$, at all three fillings, the system is in a phase where the mean field ground state is the same as that of the non-interacting case. We denote this as the symmetric phase $(S)$. The charge density is uniform and and all the symmetries of the Hamiltonian are preserved in this phase. At larger $V$, we find several charge ordered phases that break translational and rotational symmetries of the system. We characterize the charge ordering by the dipole moment $\left(P^{\mu}\right)$ and the quadrupole moment $\left(Q^{\mu \nu}\right)$ of the single particle density. These are defined as,

$$
P^{\mu} \equiv \frac{1}{N} \sum_{i} R_{i}^{\mu} \Delta_{i}
$$

$$
Q^{\mu \nu} \equiv \frac{1}{N} \sum_{i}\left(2 R_{i}^{\mu} R_{i}^{\nu}-\delta^{\mu \nu} \boldsymbol{R}_{\boldsymbol{i}} \cdot \boldsymbol{R}_{\boldsymbol{i}}\right) \Delta_{i}
$$

where $R_{i}^{\mu},(\mu=1,2)$ are the components of the position vector $\boldsymbol{R}_{\boldsymbol{i}}$ at site $i$ and $N$ is the total number of original unit cells in the lattice. It is easy to show that $Q^{\mu \nu}$ can be non-zero only if the rotational symmetry is broken and $P^{\mu}$ can be non-zero only if both rotational and inversion symmetries are broken. The phases with broken symmetry, their charge ordering and Hall conductivities are discussed below and depicted in Fig. 3. Here, the charge distribution is shown in a magnetic unit cell. The sites with same charge are shown in same color and size spheres. The charge greater in magnitude is represented with a bigger sphere. The Chern number distribution for all the filled bands in each filling is given in the bracket. The left most number in the bracket represents the Chern number for the lowest band and next number is for the next filled band. The right most number in the bracket gives the Chern number of the highest band filled.

At half filling ( $m=3$, Dirac regime), for both choices of unit cells, there is a continuous transition from the symmetric phase to a charge density wave (CDW) phase at $V=0.45$. This phase preserves all but the inversion symmetry of the system. The charge distribution in the unit cell is shown in Fig. 3. The dipole moment and quadrupole moment remain zero in this phase. As $V$ is increased, the CDW strengthens but there are no other transitions for $V \leq 10$. The Hall conductivity remains zero and the Chern number distribution in the bands remains unaltered and is similar to the $S$ phase.

For the van Hove filling, $m=2$, the energy of the mean field solution for the unit cell $\mathbf{I}$ is always lower in energy than that of II for the range of $V$ considered here. At $V=V_{c 1}=2.744$, there is a first order transition from the symmetric phase to a charge ordered nematic phase where translational and three-fold rotational symmetries are broken but the inversion symmetry of the system is intact about the center of the $A_{2} B_{2}$ links. It has non-zero quadrupole moment but the dipole moment is zero as shown in Fig. 4. Quadrupole moment is a $2 \times 2$ traceless matrix. In Fig. 4, we plot the magnitude of the eigenvalue of the quadrupole moment denoted as $|r|$. The blue line represents the plot for the magnitude of the dipole moment per original unit cell varying as a function of $V$. This Landau transition is accompanied by a topological transition where the Hall conductivity changes from $e^{2} / h$ to zero. However, the Chern numbers of the two occupied bands individually are non-zero. The Chern number of the lowest band is -1 and that of the second band is 1 (Fig. 3), hence we name the phase as the topological nematic phase $(T N)$.

As $V$ is increased, we encounter another first order transition at $V=V_{c 2}=4.5$ to a phase where the inversion symmetry is also broken. The system now develops non-zero dipole moment in addition to the quadrupole moment as shown in Fig. 4. The Chern number distribution in the occupied bands remains the same as that in 


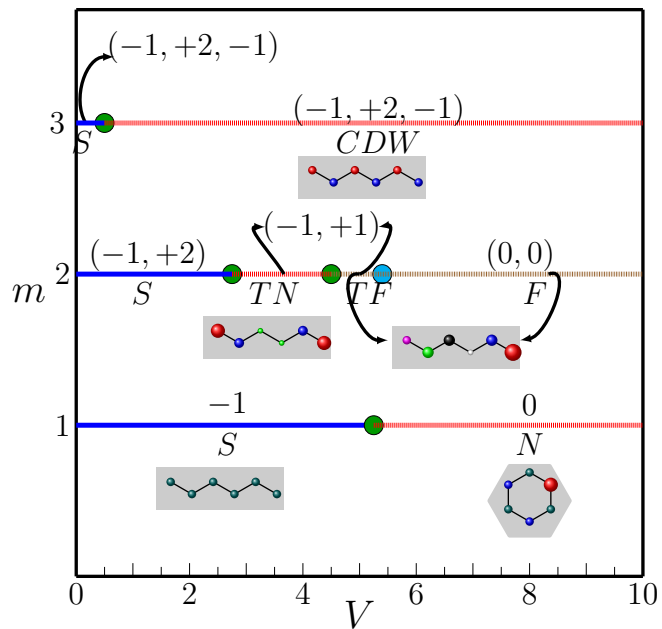

FIG. 3: (Color online) Phase diagram for $m=1,2,3$ filling as $V$ is varied. The charge distribution in each phase is shown by the unit cell whose repetition gives the full lattice picture of the ground state. The Chern number distribution of the filled bands is given in bracket. For example in the $m=2$ case, the left number in bracket is for the lowest band and the next number is for the second band. The green circle is the transition point. The blue circle is the point where the Chern numbers of the filled bands change without any change in the Hall conductivity.

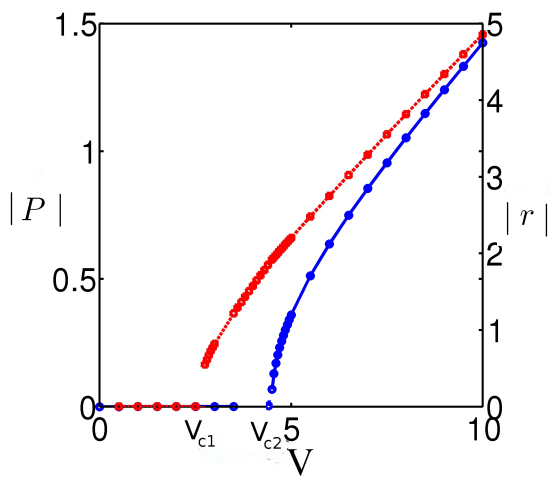

FIG. 4: (Color online) The quadrupole and dipole moments as a function of $V$ in the three phases for $m=2$. The blue line represents the polarization (i.e. dipole moment per original unit cell)and the red line represents the magnitude of the eigenvalue of the quadrupole moment matrix per original unit cell, $|r|$.

the $T N$ phase (Fig. 3). We call this phase, the topological ferri-electric phase $(T F)$. The two occupied bands touch at $V=5.41$ and there is a redistribution of Chern numbers between these bands. For $V>5.41$ the Chern number of each of the filled bands become zero (Fig. 3). This phase is denoted as the ferri-electric phase $(F)$.

In the Fermi regime, $m=1$, the energy for the mean field solution with unit cell choice II is lower than that of $\mathbf{I}$. Here, we find a first order transition from the symmetric phase to a nematic phase $(N)$ (Fig. 3), similar to that described for $m=2$, at $V=5.265$. The charge distribution has no dipole moment but has nonzero quadrupole moment. The Hall conductivity changes from $\sigma_{H}=-e^{2} / h$ to zero at this transition. Thus, this is also a first order Landau transition accompanied by a topological transition.

The charge ordered phases with broken translational and three-fold rotational symmetries also have anisotropic bond order parameters, $\chi_{\langle(\alpha, a)(\beta, b)\rangle}$. The anisotropic magnitudes of the order parameters are shown in Fig. 6 for $m=1$ and 2. For $m=2$, the bond order parameters also acquire anisotropic phases which manifest as circulating currents.
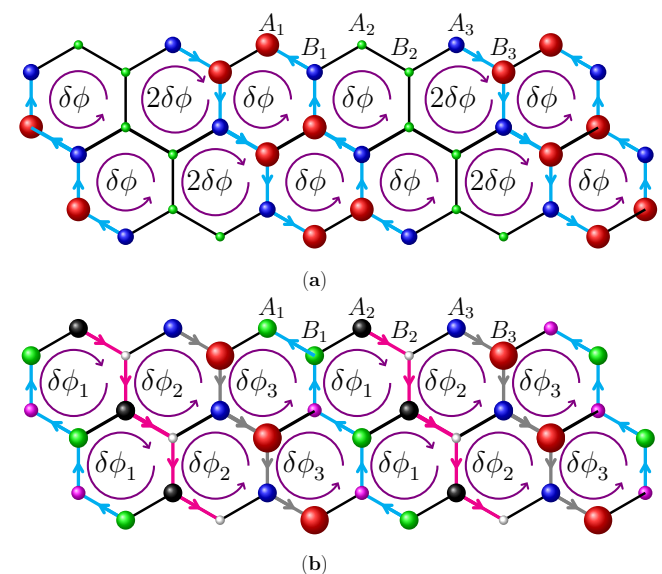

FIG. 5: (Color online) Flux in the plaquette and current flowing on the links on the lattice for $m=2$ in (a) nematic phase (b) ferri-electric phase. The flux distribution is shown after subtracting out the background flux $2 \pi / 3$. In ferri-electric phase, $\delta \phi_{1}=\delta \phi_{3}+\delta \phi_{2}$. The bonds with arrows are the links on which there is non-zero current. Same color represents same magnitude of current.

There is no current on the links in the symmetric phase. Fig. 5 shows the current on the links on the lattice in nematic and ferri-electric phases. In nematic phase, currents of equal magnitude flow on the links $A_{1} B_{1}$ and $A_{3} B_{3}$ (links with arrows) in opposite direction (as shown in Fig. 5a) such that the inversion symmetry (about $A_{2} B_{2}$ link) of the system is preserved. There is no current on $A_{2} B_{2}$ links. In ferri-electric phase, the current flows on the links $A_{1} B_{1}, A_{2} B_{2}$ and $A_{3} B_{3}$ (Fig. 5b) such that the total current on the links in the magnetic unit cell is zero. The current distribution shows that the inversion symmetry of the system along with the rotational symmetry is broken. This figure also gives the distribution of flux in the plaquettes of the system in nematic and ferri-electric phases. In Fig. 5 the flux distribution is shown after subtracting out the background flux $2 \pi / 3$. As seen in Fig. 5a, the staggered flux in the nematic phase is distributed in a way that the inversion 
symmetry of the lattice is preserved unlike that in the ferri-electric phase where the flux distribution shows inversion symmetry breaking. In both the cases the total flux in the magnetic unit cell is zero.

We now compare these staggered flux patterns in the lattice with those discussed by Castro et al. ${ }^{10}$, where they analyzed the system without a magnetic field. In their paper, they have described about two interaction induced spontaneously time reversal symmetry (TRS) broken phases, T-I and T-II phases. While in T-II phase the inversion symmetry is preserved, T-I phase breaks the inversion symmetry. We see that the flux pattern for nematic phase is similar to that of their T-II phase. In both these cases inversion symmetry of the system is preserved. The staggered flux pattern in ferri-electric phase shows inversion symmetry breaking in the system as is the case for T-I phase in ${ }^{10}$; However, in ferri-electric phase every plaquette has non-zero flux passing through it and the staggered flux in all the three plaquettes forming the magnetic unit cell have different magnitude unlike the T-I phase. The current on the links in our system form a stripe pattern unlike the case without the magnetic field ${ }^{10}$ where the current on the links form closed loops.

To try and get some insight to the mechanism of the topological transitions accompanying the Landau transitions, we examine the pattern of the anisotropic magnitudes of the bond order parameters shown in Fig. 6. The bond strength distribution for the $T N, T F$ and $F$ phases indicate that the mean-field Hamiltonian resembles that of weakly coupled ribbons, namely a quasi-1d system. The coupling becomes weaker as the interaction strength is increased. In the limit of completely decoupled ribbons, Chern numbers for all the bands can be equal to zero and this happens for $V>5.41$. Thus it seems that the anisotropy of the bond order parameters drives the change of Chern numbers. The $N$ phase at $m=1$ is similar except that it tends to a system of weakly coupled clusters as shown in Fig. 6b.

However, while we believe that there is some truth to the argument given above, there are some caveats. Firstly, the transitions need not happen at a finite value of $V$ but could happen only at $V=\infty$. Secondly when the system gets decoupled, the Chern numbers of the individual bands can get ill-defined due to degeneracies. Both these issues can be illustrated in the half-filled case of $m=3$. As shown in Fig. 3, the Chern numbers in this case remain unchanged throughout the range, $0 \leq V \leq 10$. We now show that this is true for arbitrary $V$. As mentioned earlier, the single particle mean field Hamiltonian is of the form, $h_{M F}(\vec{k})=h_{0}(\vec{k})+h_{F}(\vec{k}, \chi, \Delta)$ For the half filled case, the system is isotropic and the bond order parameters simply scales $h_{0}(\vec{k})$ and the charge modulation is $\pm \Delta$ for the two sublattices. Since $h_{0}(\vec{k})$ does not couple the two sublattices, we can write $\vec{h}_{M F}(\vec{k})$ as,

$$
h_{M F}(\vec{k})=\left(\begin{array}{cc}
\Delta & F(\vec{k}) \\
F^{\dagger}(\vec{k}) & -\Delta
\end{array}\right)
$$

It can be proved, as we do in Appendix A, that the Chern numbers of the above Hamiltonian are independent of $\Delta$. As $V \rightarrow \infty$ we also have $\Delta \rightarrow \infty$. In the limit, there are two degenerate ground state corresponding to all the particles occupying one of the sublattices. However, all the the negative energy single particle states are degenerate and thus the three lower bands are completely degenerate. Thus the individual Chern numbers are ill-defined at $V=\infty$. At $\Delta=0$ (the symmetric phase), the middle band Chern numbers are ill-defined due to the Dirac points. However, the system is particle-hole symmetric in this phase and the Chern numbers get fixed by demanding that the sum of the Chern numbers, which is proportional to the Hall conductivity, is zero. Thus the Chern numbers in the half filled case are unchanged in the range $0 \leq V<\infty$.
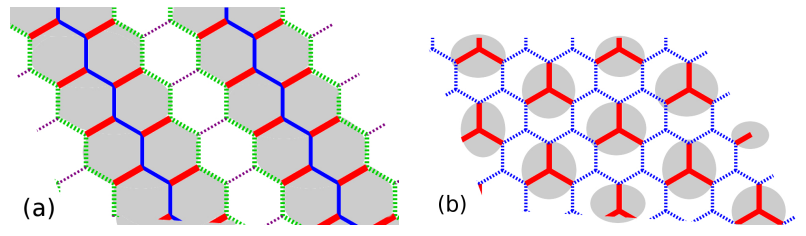

FIG. 6: (Color online) (a) Bond order parameter strengths for $m=2$ broken symmetry phases. The red bonds are the strongest and the dotted violet bonds the weakest. (b) Bond order parameters for the $m=1$ broken symmetry phase. The red bonds are stronger than the dotted blue bonds.

\section{GEOMETRY OF THE GROUND STATES}

The structure of incompressible liquids is described by the pair correlation function. For our system it is defined as,

$$
\Gamma_{\alpha a, \beta b}\left(\boldsymbol{R}_{I}-\boldsymbol{R}_{J}\right)=\left\langle c_{I a \alpha}^{\dagger} c_{J b \beta}^{\dagger} c_{J b \beta} c_{I a \alpha}\right\rangle
$$

where $I, J$ represent the position of the magnetic unit cell. The anisotropic state of the system can be understood in terms of this pair correlation function. The nematic order parameter has been related to the quantum metric ${ }^{20}$. In this section, we would therefore like to study the pair correlation function in the light of quantum geometry described by the quantum metric. Resta $^{25}$ has derived an exact relationship between the momentum space quantum metric ${ }^{24}$ averaged over the $\mathrm{BZ}, \bar{g}^{\mu \nu}$, and the pair correlation function, more precisely, to the structure factor 


$$
\begin{array}{r}
\bar{g}^{\mu \nu}=\frac{1}{L^{2}} \sum_{I \alpha a, J \beta b}\left(R_{I \alpha a}^{\mu}-R_{J \beta b}^{\mu}\right)\left(R_{I \alpha a}^{\nu}-R_{J \beta b}^{\nu}\right) \\
S_{\alpha a, \beta b}\left(\boldsymbol{R}_{I}-\boldsymbol{R}_{J}\right),
\end{array}
$$

where $L^{2}$ is the area of the system and $S_{\alpha a, \beta b}\left(\boldsymbol{R}_{I}-\boldsymbol{R}_{J}\right)=$ $\left\langle c_{I \alpha a}^{\dagger} c_{I \alpha a}\right\rangle\left\langle c_{J \beta b}^{\dagger} c_{J \beta b}\right\rangle-\Gamma_{\alpha a, \beta b}\left(\boldsymbol{R}_{I}-\boldsymbol{R}_{J}\right)$.

Thus $\bar{g}^{\mu \nu}$ is the second moment of the structure factor. It therefore characterizes the shape of the pair correlation function just as the real space metric introduced by Haldane $^{18}$ for homogeneous quantum Hall systems. To obtain a more precise relation between the two, we need to examine the weak field (large $q$ ) limit. We postpone this for future work.

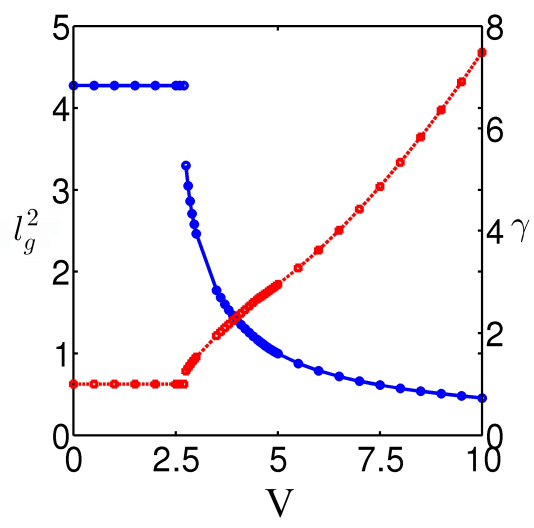

FIG. 7: (Color online) $l_{g}^{2}=\sqrt{g_{1} g_{2}}$ and $\gamma$ vs $V$ for $m=2$. The blue line represents $l_{g}$ and the red line represents $\gamma$. The discontinuity in the plot shows the first order nature of the phase transition.

The metric is a second rank symmetric tensor and has three independent components. These can be taken to be the orientation of the principle axis, $\theta$ and the two eigenvalues $g_{1}, g_{2}$. Eq. (8) shows that the spread of the pair correlation function in the directions along and normal to the principal axis is given by $\sqrt{g_{1}}$ and $\sqrt{g_{2}}$. Therefore, the areal extent of the correlations $\sim \sqrt{g_{1} g_{2}} \equiv l_{g}^{2}$, the square root of the determinant of the $\bar{g}^{\mu \nu}$. The ratio of the two eigenvalues $\gamma=g_{1} / g_{2}$ is a measure of its anisotropy. $l_{g}^{2}$ and $\gamma$ are plotted as a function of the interaction strength in Fig. 7. $l_{g}^{2}$ decreases while the anisotropic parameter $\gamma$ increases on increasing $V$.

The structure function reflects the symmetry (or the lack of it) of the ground state. Fig. 8a shows the structure function plotted in the real space for symmetric phase for $m=2 . \quad R_{x}$ and $R_{y}$ give the position of the lattices in the real space in Cartesian coordinates. We see that in this plot, the pair correlation function is invariant under $2 \pi / 3$ rotation since the symmetric phase preserves the rotational symmetry of the system. Fig. $8 \mathrm{~b}$ shows the structure function plotted in the real space for nematic phase for $m=2$. In this plot, the structure function is not invariant under $2 \pi / 3$ rotation since in nematic phase the rotational symmetry is broken.

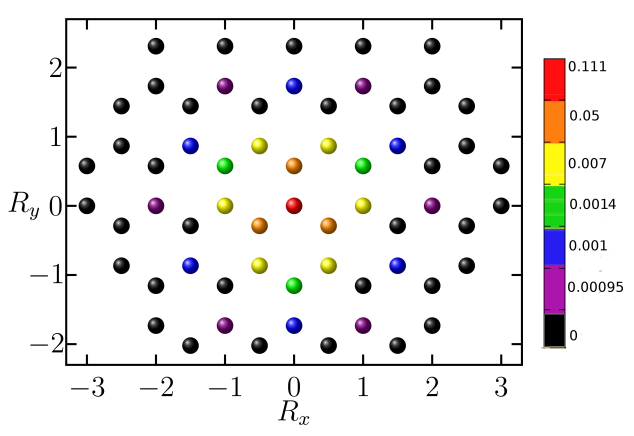

(a)

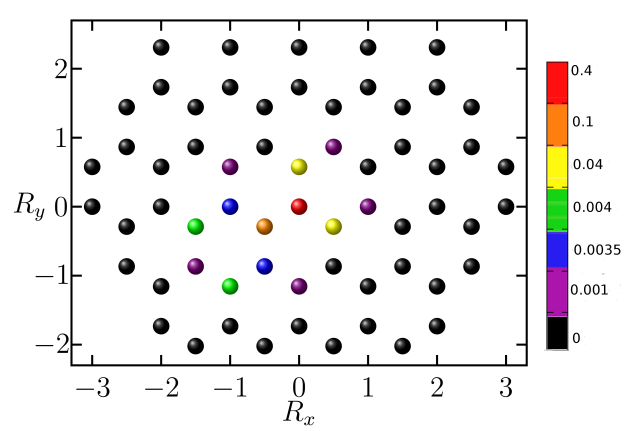

(b)

FIG. 8: (Color online) Pair correlation function on lattice in (a) symmetric phase showing $2 \pi / 3$ rotation preserved, (b) nematic phase for $m=2$ showing $2 \pi / 3$ rotation broken.

The average metric is a multiple of identity in the symmetric phase. In the nematic and ferri-electric phases it becomes anisotropic, with its principle axis aligned with one of the basis vectors.

\section{CONCLUSION}

To summarize our results, we show that the nearest neighbor repulsive interaction induces a charge ordering, as we intuitively expect. At strong interactions, translation symmetry broken, anisotropic charge distributions become energetically favorable. The anisotropy in the particle density can be characterized by the quadrupole and dipole moments. The anisotropy and the spatial extent of the pair correlations are characterized by the quantum metric averaged over the BZ.

The first order transition for $m=1,2$ from the symmetric to the nematic phase is accompanied with change of topology which is reflected in the change in the Hall conductivity. Though the Hall conductivity is zero in the nematic phase, in $m=2$ band, the filled bands individually have non-trivial topology with non-zero Chern number. Some insight for the mechanism of this transition comes from examining the magnitudes of the bond order parameters, $\chi_{\langle\alpha a, \beta b\rangle}$. The $2 \mathrm{~d}$ lattice looks like a set of weakly coupled $1 \mathrm{~d}$ ribbons or clusters. In the ex- 
treme limit of decoupled ribbons, the Chern number is zero and hence will remain so for weak coupling as well. So a Chern number change accompanies the first order nematic transition when the weakening is significant.

In conclusion, our results show that interactions induce interesting and complex phases in the Hofstadter regime of the honeycomb lattice.

The fractal structure is understood as arising from the interplay between the two independent length scales, the periodicity and the magnetic length. We have shown that the interaction induces charge ordering which breaks the translational symmetry and thus changes the periodicity. The effect of this change of the periodicity on the fractal structure of the Hofstadter butterfly requires a study of the translation symmetry breaking pattern for other values of $\phi / \phi_{0}$ as well. This work is in progress.

\section{Acknowledgments}

We are grateful to A. M. M. Pruisken, Dibyakrupa Sahoo, Jainendra K. Jain, Mukul S. Laad, S. Arya and Vinu Lukose for useful discussions.

\section{Appendix A: Chern numbers at half filling}

The single particle mean field Hamiltonian for the half filled case describing the CDW state is given in equation (6). The eigenvalue equation can be written as,

$$
\left(\begin{array}{cc}
\Delta & F(\vec{k}) \\
F^{\dagger}(\vec{k}) & -\Delta
\end{array}\right)\left(\begin{array}{c}
\psi_{A}(\vec{k}) \\
\psi_{B}(\vec{k})
\end{array}\right)=E(\vec{k})\left(\begin{array}{c}
\psi_{A}(\vec{k}) \\
\psi_{B}(\vec{k})
\end{array}\right)
$$

where $\psi_{A(B)}(\vec{k})$ are $q$-component column vectors. They can be constructed in terms of the spectrum of the positive, semi-definite, hermitian matrix, $F(\vec{k}) F^{\dagger}(\vec{k})$. We denote,

$$
F(\vec{k}) F^{\dagger}(\vec{k}) \chi^{n}(\vec{k})=\epsilon_{n}^{2}(\vec{k}) \chi^{n}(\vec{k})
$$

where, $n=1, \ldots, q$ and we choose $\chi^{n}$ to be orthonormalized. The above equation implies that the eigenvalues of $F^{\dagger}(\vec{k}) F(\vec{k})$ are the same, since,

$$
F^{\dagger}(\vec{k}) F(\vec{k})\left(F^{\dagger}(\vec{k}) \chi^{n}(\vec{k})\right)=\epsilon_{n}^{2}(\vec{k})\left(F^{\dagger}(\vec{k}) \chi^{n}(\vec{k})\right)
$$

Further, the inversion (two fold rotation) transformation relates $F(\vec{k})$ to $F^{\dagger}(-\vec{k})$,

$$
\mathcal{I} F(\vec{k}) \mathcal{I}^{\dagger}=F^{\dagger}(-\vec{k})
$$

where,

$$
\mathcal{I}=\left(\begin{array}{lll}
0 & 0 & 1 \\
0 & 1 & 0 \\
1 & 0 & 0
\end{array}\right)
$$

consequently, we have $\epsilon_{n}(\vec{k})=\epsilon_{n}(-\vec{k})$ and

$$
F^{\dagger}(\vec{k}) F(\vec{k})\left(\mathcal{I} \chi^{n}(-\vec{k})\right)=\epsilon_{n}^{2}(\vec{k})\left(\mathcal{I} \chi^{n}(-\vec{k})\right)
$$

The eigenvectors of $h_{M F}(\vec{k}), \psi^{ \pm n}(\vec{k})$ corresponding to the eigenvalues $E_{ \pm n}(\vec{k})= \pm \sqrt{\epsilon_{n}^{2}(\vec{k})+\Delta^{2}}$ are given by,

$$
\begin{aligned}
& \psi^{+n}(\vec{k})=\left(\begin{array}{c}
\cos \frac{\theta_{n}(\vec{k})}{2} \chi^{n}(\vec{k}) \\
\sin \frac{\theta_{n}(\vec{k})}{2} \mathcal{I} \chi^{n}(-\vec{k})
\end{array}\right) \\
& \psi^{-n}(\vec{k})=\left(\begin{array}{c}
-\sin \frac{\theta_{n}(\vec{k})}{2} \chi_{n}(\vec{k}) \\
\cos \frac{\theta_{n}(\vec{k})}{2} \mathcal{I} \chi^{n}(-\vec{k})
\end{array}\right)
\end{aligned}
$$

where,

$$
\cos \theta_{n}(\vec{k})=\frac{\Delta}{\sqrt{\epsilon_{n}^{2}(\vec{k})+\Delta^{2}}}, \quad \sin \theta_{n}(\vec{k})=\frac{\epsilon_{n}(\vec{k})}{\sqrt{\epsilon_{n}^{2}(\vec{k})+\Delta^{2}}}
$$

The Pancharatnam-Berry curvatures of the negative energy bands (occupied at half filling) are given by $\mathcal{B}^{-n}(\vec{k})=\epsilon_{i j} \partial_{i} \psi^{-n^{\dagger}}(\vec{k}) \partial_{j} \psi^{-n}(\vec{k})$. The Chern numbers are given by,

$$
\begin{aligned}
\nu^{n}= & \int_{k}\left(\epsilon_{i j} \sin \theta_{n} \partial_{i} \theta_{n}\left(\tilde{\mathcal{A}}_{j}^{n}(\vec{k})+\tilde{\mathcal{A}}_{j}^{n}(-\vec{k})\right)+\right. \\
& \left.\epsilon_{i j}\left(\sin ^{2} \frac{\theta_{n}}{2} \tilde{\mathcal{B}}^{n}(\vec{k})+\cos ^{2} \frac{\theta_{n}}{2} \tilde{\mathcal{B}}^{n}(-\vec{k})\right)\right)(
\end{aligned}
$$

where the integral is over the reduced BZ, $\tilde{\mathcal{A}}_{i}^{n}(\vec{k}) \equiv$ $-i \chi^{n \dagger}(\vec{k}) \partial_{i} \chi^{n}(\vec{k})$ and $\tilde{\mathcal{B}}^{n}(\vec{k})=\epsilon_{i j} \partial_{i} \tilde{\mathcal{A}}^{n}(\vec{k})$. Since, $\tilde{\mathcal{A}}_{i}^{n}(\vec{k})=-\tilde{\mathcal{A}}_{i}^{n}(-\vec{k}), \quad \tilde{\mathcal{B}}^{n}(\vec{k})=\tilde{\mathcal{B}}^{n}(-\vec{k})$ and $\theta_{n}(\vec{k})=$ $\theta_{n}(-\vec{k})$, we get our final result,

$$
\nu^{n}=\int_{k} \tilde{\mathcal{B}}^{n}(\vec{k})
$$

Thus, since $\chi^{n}(\vec{k})$ is independent of $\Delta$, so are the Chern numbers.

\section{Appendix B: Energy Band Diagram}

Fig. 9a and Fig. 9b is the energy band diagram for $q=3$ at $m=2$ filling in the symmetric and nematic phase respectively at the transition point $V=2.744$. We see that in Fig. 9a, $E_{k_{1}, k_{2}}=E_{k_{1}, k_{2}+2 \pi / 3}$ which is the result of the system being invariant under the translational symmetry unlike in the nematic phase where translational symmetry is broken which is reflected in the energy band diagram, Fig. $9 \mathrm{~b}$, where $E_{k_{1}, k_{2}} \neq E_{k_{1}, k_{2}+2 \pi / 3}$. Hence, in the later case, the length scale, the periodicity of the mean field Hamiltonian, is now same as that of the periodicity of the magnetic unit cell. So the ground state and the periodicity depends on the magnetic field. As seen in Fig. 9a and Fig. 9b, there is always a band gap 


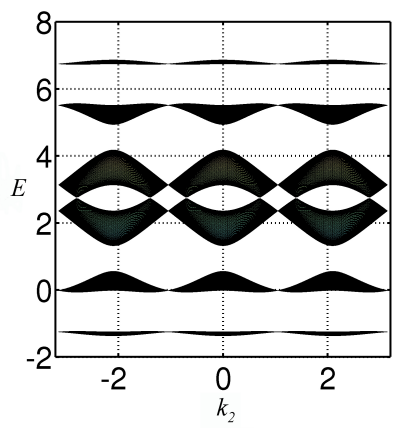

(a)

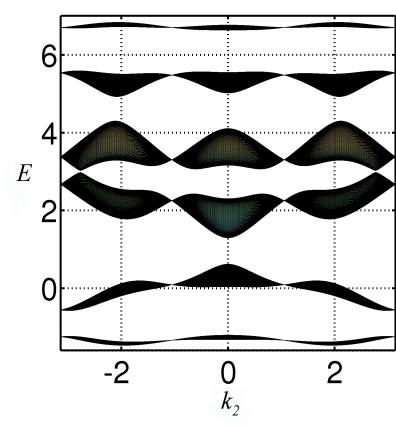

(b)
FIG. 9: (Color online) Energy band diagram w.r.t $k_{2}$ for (a) $q=3$ in symmetric phase for $m=2$ at $V=2.744$, (b) $q=3$ in nematic phase for $m=2$ at $V=2.744$. The energy band diagrams are plotted by diagonalizing the mean field Hamiltonian using the order parameters obtained by solving the self consistency equations for $m=2$ and $q=3$. between the filled bands and the first excited band (i.e. band gap between between second and third bands). The bandwidth of the bands increases in the translation symmetry broken phase and the band gap decreases but is never zero in the interaction strength considered. Therefore, the mean field approximation is a good approximation here.
1 D. R. Hofstadter, Physical review B 14, 2239 (1976).

2 L. Ponomarenko, R. Gorbachev, G. Yu, D. Elias, R. Jalil, A. Patel, A. Mishchenko, A. Mayorov, C. Woods, J. Wallbank, et al., Nature 497, 594 (2013).

3 C. Dean, L. Wang, P. Maher, C. Forsythe, F. Ghahari, Y. Gao, J. Katoch, M. Ishigami, P. Moon, M. Koshino, et al., Nature 497, 598 (2013).

${ }^{4}$ B. Hunt, J. Sanchez-Yamagishi, A. Young, M. Yankowitz, B. J. LeRoy, K. Watanabe, T. Taniguchi, P. Moon, M. Koshino, P. Jarillo-Herrero, et al., Science 340, 1427 (2013).

${ }^{5}$ G. Yu, R. Gorbachev, J. Tu, A. Kretinin, Y. Cao, R. Jalil, F. Withers, L. Ponomarenko, B. Piot, M. Potemski, et al., Nature Physics 10, 525 (2014).

${ }^{6}$ G. Yu, R. Gorbachev, J. Tu, A. Kretinin, Y. Cao, R. Jalil, F. Withers, L. Ponomarenko, B. Piot, M. Potemski, et al., Nature Physics 10, 784 (2014).

7 M. Aidelsburger, M. Atala, M. Lohse, J. T. Barreiro, B. Paredes, and I. Bloch, Physical review letters 111, 185301 (2013).

${ }^{8}$ H. Miyake, G. A. Siviloglou, C. J. Kennedy, W. C. Burton, and W. Ketterle, Physical review letters 111, 185302 (2013).

9 C. Weeks, and M. Franz, Physical Review B 81, 085105 (2010).

10 E. V. Castro, A. G. Grushin, B. Valenzuela, M. A. H. Vozmediano, A. Cortijo, and F. de Juan, Physical review letters 107, 106402 (2011).

11 A. G. Grushin, E. V. Castro, A. Cortijo, F. de Juan, M. A. H. Vozmediano, and B. Valenzuela, Physical Review B 87, 085136 (2013).
12 V. Gudmundsson and R. R. Gerhardts, Physical Review B 52, 16744 (1995).

${ }^{13}$ H. Doh and S. Sung-HoSuck Salk, Phys. Rev. B 57, 1312 (1998).

14 K. Czajka, A. Gorczyca, M. M. Maśka, and M. Mierzejewski, Physical Review B 74, 125116 (2006).

15 T. Chakraborty, and V. M. Apalkov Solid State Communications 175-176, 123 (2013).

16 V. M. Apalkov and T. Chakraborty, Physical review letters 112, 176401 (2014).

17 A. A. Koulakov, M. M. Fogler, and B. I. Shklovskii, Physical review letters 76, 499 (1996).

18 F. D. M. Haldane, Physical review letters 107, 116801 (2011).

19 Y. You, G. Y. Cho, and E. Fradkin, Physical Review X 4, 041050 (2014).

20 J. Maciejko, B. Hsu, S. A. Kivelson, Y. J. Park, and S. L. Sondhi, Physical Review B 88, 125137 (2013).

21 M. P. Lilly, K. B. Cooper, J. P. Eisenstein, L. N. Pfeiffer, and K. W. West, Physical Review Letters 82, 394 (1999).

22 J. Xia, J. Eisenstein, L. Pfeiffer, and K. West, Nature Physics 7, 845 (2011).

23 Y. Liu, S. Hasdemir, M. Shayegan, L. N. Pfeiffer, K. W. West, and K. W. Baldwin, Physical Review B 88, 035307 (2013).

24 R. Resta, The European Physical Journal B-Condensed Matter and Complex Systems 79, 121 (2011).

25 R. Resta Physical Review Letters 96, 137601 (2006).

26 Y. Hatsugai, T. Fukui, and H. Aoki, Phys. Rev. B 74, 205414 (2006). 